\title{
BRST Gauge Fixing and Regularization
}

\author{
P.H. DAMGAARD* \\ Institute of Theoretical Physics, Uppsala University, S-751 08 Uppsala, Sweden \\ F. DE JONGHE \\ NIKHEF-H, Postbus 41882, 1009 DB Amsterdam, The Netherlands \\ and \\ R. SOllacher \\ Institute of Theoretical Physics, Uppsala University, S-751 08 Uppsala, Sweden
}

July 23, 2018

\begin{abstract}
In the presence of consistent regulators, the standard procedure of BRST gauge fixing (or moving from one gauge to another) can require non-trivial modifications. These modifications occur at the quantum level, and gauges exist which are only well-defined when quantum mechanical modifications are correctly taken into account. We illustrate how this phenomenon manifests itself in the solvable case of two-dimensional bosonization in the path-integral formalism. As a by-product, we show how to derive smooth bosonization in Batalin-Vilkovisky Lagrangian BRST quantization.
\end{abstract}

UUITP-8/95

NIKHEF-H 95-017

hep-th/9505040

* On leave of absence from the Niels Bohr Institute, Blegdamsvej 17, DK-2100 Copenhagen, Denmark. 
1. Essentially all approaches to gauge fixing in the path integral formalism are based on formal manipulations that ignore the issue of regularization. It is known, moreover, that care may be required if one is to work in the framework of a (perturbatively) regularized quantum field theory. For a recent discussion of this issue in the context of Yang-Mills theory, see ref. [1].

Actually, the problem of a suitable BRST gauge-fixing procedure in the regularized path integral goes even deeper. As we shall describe in this letter, there are a number of situations in regularized Lagrangian quantum field theory where at least three known methods of gauge fixing (FaddeevPopov [2], usual Lagrangian BRST [3], and Batalin-Vilkovisky [4]) all fail to produce a correctly gauge-fixed path integral, when applied naively. We shall also show how solutions to this problem can be found.

Consider a gauge theory based on an action $S[\phi]$, and a local gauge transformation $\delta \phi^{i}=$ $R_{\alpha}^{i}[\phi] \epsilon^{\alpha}$. Suppose that under this gauge transformation a certain operator $\mathcal{O}$ remains invariant at the classical level, but is modified at the quantum level. Obviously, at the classical level the operator $\mathcal{O}$ will be unsuitable for a gauge-fixing function. Since gauge fixing refers not just to the classical aspects of the field theory but also to the quantum level, a natural question to ask is the following: can one gauge-fix on the operator $\mathcal{O}$ in the full theory, which incorporates quantum effects? This problem is not nearly as contrived as it may appear at first sight. It arises in the 2-dimensional context of "smooth bosonization" if one follows the path of ref. [5] and in the general discussion of an effective $U(1)$ flavour-singlet Lagrangian using collective fields [6]. In both of these examples, one essentially has to "gauge fix on the chiral anomaly", and for this reason the solution to that particular gauge-fixing problem was called "anomalous gauge fixing". But the problem appears in related contexts even when the operator in question is not anomalous, only suffers a certain (non-anomalous) modification at the quantum level [7]. In refs. [5, 6, 7] various valid solutions to this rather unusual gauge-fixing problems were presented, but no general procedure was outlined. We emphasize again that modifications to the naive rules of (BRST) gauge fixing are required in all of these cases, and that these modifications are inescapable if one wishes to correctly describe the quantum mechanical phenomena in those gauges. . An example of an analogous gauge-fixing anomaly within the context of string theory is discussed in ref. [8].

Clearly, the issue of an operator being invariant at the classical level, but undergoing modifications due to quantum corrections, is intimately tied to the choice of regulator. If we abandon the operator language by working instead in the Lagrangian path integral formalism, the "operators" remain unchanged when manipulated inside the functional integral, but the analogues of the quantum corrections show up due to effects of the regulators. It is here convenient to consider regularization in a (generalized) Pauli-Villars sense. At the one-loop level, an explicit correspondence between this kind of regularization and a Fujikawa-type [9] path-integral regularization of the measure can be derived [10]. At the one-loop level we can thus consider the whole regularization as arising from the path integral measure. It is important to keep in mind that in general consistent regularizations of the path integral measure will depend non-trivially on the action $S[\phi]$ under consideration. From the point of view that the regularized path-integral measure can be obtained at the one-loop level from a particular Pauli-Villars scheme by integrating out regularizing "Pauli-Villars fields" (using suitable integration rules [10]), this is of course entirely natural.

If the regularized path integral measure remains invariant under the local gauge transformation and the associated global BRST transformation, then no problems of the kind discussed above can arise. This is obvious, since (at least to the one-loop level at which we are restricting ourselves for the moment) no operators $\mathcal{O}$ that are classically gauge (or BRST) invariant do not remain invariant at the quantum level. It does not mean, however, that gauge fixing - or in a more precise language:

\footnotetext{
${ }^{1}$ In some special cases a way to circumvent the problem can be found. See below.
} 
once fixed, going from one gauge to another - is an entirely trivial matter. In the usual Lagrangian BRST formalism going from one gauge to another can be achieved by adding a "gauge fermion" of the type $\delta \Psi[\phi]$ (where $\delta$ here refers to the associated BRST variation) to the previous action:

$$
S[\phi] \rightarrow \tilde{S}[\phi]=S[\phi]+\delta \Psi[\phi] .
$$

Clearly, adding this new term will in general affect the needed regularization as well. So the functional measure must be modified appropriately:

$$
\int[d \phi]_{S} \exp \left[\frac{i}{\hbar} S[\phi]\right] \rightarrow \int[d \phi]_{\tilde{S}} \exp \left[\frac{i}{\hbar}\{\tilde{S}[\phi]+\delta \Psi[\phi]\}\right]
$$

where $\phi^{A}(x)$ stands for the generic collection of classical fields $\phi^{i}$, ghosts, antighosts, auxiliary fields, ghosts-for-ghosts, etc., as needed. This required modification of the functional measure simultaneously with adding gauge fermions is the source of new (and often surprising) effects that may arise when the gauge-fixing operators transform differently at the classical and quantum level.

Consider now the possibility that neither the action nor the measure, but only the combination of the two, is invariant under the BRST transformation. To the one-loop level this means that the partition function must be of the form

$$
Z=\int[d \phi]_{S} \exp \left[\frac{i}{\hbar}\left\{S[\phi]+\hbar \mathcal{M}_{1}[\phi]\right\}\right],
$$

where the BRST variation $i \delta \mathcal{M}_{1}$ precisely cancels the logarithm of the Jacobian. Non-invariance of the measure can arise either as a result of the regularization, or be inherent to the formalism, as in, e.g., the case of open gauge algebras. There may or may not be higher order contributions (in $\hbar$ ) to the action, and we shall return to this more general all-order situation below, but for the present a one-loop consideration will suffice. Notice that the measure is regularized only with respect to $S$ (and not $S+\hbar \mathcal{M}_{1}$ ), since either $\mathcal{M}_{1}$ does not affect the regularization, or, if it does, the effect of modifying the measure on account of this will be of higher order in $\hbar$. What happens now when we add the BRST-variation of a gauge fermion? Formally, i.e., when ignoring issues of regularisation, adding a term of the form $\delta \Psi[\phi]$ to the action does not affect BRST invariance of the functional integral (since $\delta^{2}=0$ ), and does not affect the expectation values of BRST-invariant observables. Let us try the same procedure in the regularized path integral (3):

$$
\int[d \phi]_{S} \exp \left[\frac{i}{\hbar}\left\{S[\phi]+\hbar \mathcal{M}_{1}[\phi]\right\}\right] \rightarrow \int[d \phi]_{S+\delta \Psi} \exp \left[\frac{i}{\hbar}\left\{S[\phi]+\hbar \mathcal{M}_{1}[\phi]+\delta \Psi[\phi]\right\}\right] .
$$

The r.h.s. of (đ) does not in general describe a BRST-symmetric path integral. This is due to the fact that the cancellation between the measure and $i \mathcal{M}_{1}$ in general will be destroyed, since the Jacobian from the measure is $\delta \Psi$-dependent, while $\mathcal{M}_{1}$ is not. So the first lesson we learn is that in the functional integral, when regularized through its measure, adding BRST-exact terms to the action may break the BRST symmetry.2

At the one-loop level an obvious cure to this problem presents itself. When we add (or change) a gauge fermion through a term $\delta \Psi$, we can correct the non-invariance of the measure and action by re-calculating the one-loop counterterm $i \hbar \mathcal{M}_{1}$ by using the new regularized measure. The final result will then by construction be BRST invariant to the one-loop level. The only unpleasant by-product of this procedure is that we seem to lose some of the power of gauge fixing. Knowing

\footnotetext{
${ }^{2}$ If one insists that the path integral shall remain properly regularized after the addition of the BRST-exact term to the action. One can keep BRST symmetry at the cost of losing the proper regularization, but we discard this possibility.
} 
which gauge we are actually going to by adding a term $\delta \Psi$ will now require a one-loop calculation, and cannot be read off immediately from the original action. An alternative route can be found by keeping the regulator fields in the action throughout, and by requiring that the gauge fermion $\Psi$ be replaced by a properly regularized $\Psi_{R}$. The whole procedure is then again by construction BRST invariant and properly regularized. As we shall illustrate below, it will however, in general lead to rather unusual gauge-fixings in terms of the original fields. It will also typically lead to higher-order terms in the ghost fields, once the regulator fields are integrated out of the path integral.

2. Let us illustrate these last considerations by the example of a two-dimensional field theory of Dirac fermions coupled to external Abelian axial sources [5]. The Pauli-Villars-regularized version reads

$$
\begin{aligned}
Z[A] & =\int d[\bar{\psi}, \psi] \prod_{l} d\left[\bar{\psi}_{l}, \psi_{l}\right] \exp \left(\frac{i}{\hbar} S_{0}\right) \\
S_{0} & =\int d^{2} x\left[\bar{\psi} i \not D \psi+\sum_{l} \bar{\psi}_{l}\left(i \not D-M_{l}\right) \psi_{l}\right]
\end{aligned}
$$

with $D_{\mu}=\partial_{\mu}-i A_{\mu} \gamma_{5}$. We choose a regularization scheme such that the following relations hold (cf. ref. [11):

$$
\sum_{l} c_{l}=1 \quad, \quad \sum_{l} c_{l} k_{l}^{m}=0 \text { for } m=1,2 \quad, \quad M_{l}=k_{l} \Lambda
$$

The regulator masses are proportional to a cutoff $\Lambda$ which may eventually be sent to infinity. For simplicity we take the coefficients $c_{l}$ to be +1 or -1 depending on whether the corresponding regulator fields are Grassmann-even or -odd. Although this may look like a free theory, the coupling to the sources makes it non-trivial, and by e.g. integrating over these sources with appropriate measures, we can easily turn it into a genuinely interacting theory. For this reason we need to regularize the path integral by a suitable number of Pauli-Villars fields, here labelled by $l$. The measures of all the fields are otherwise unconstrained, because the path integral regularization is completely taken into account (up to one loop order) by the regulator fields $\psi_{l}$. This system can conveniently be viewed as an Abelian gauge theory in disguise. The missing gauge degree of freedom can be re-introduced through a field redefinitions of the original fields $\bar{\psi}, \psi$. We follow the example of [5] and choose a chiral transformation

$$
\bar{\psi}=\bar{\chi} e^{i \gamma_{5} \theta} \quad, \quad \psi=e^{i \gamma_{5} \theta} \chi,
$$

which introduces a new pseudoscalar field $\theta(x)$. Because we regularize the theory by explicit PauliVillars fields, and because we wish to deal only with properly regularized expressions, we let the regulator fields transform analogously:

$$
\bar{\psi}_{l}=\bar{\chi}_{l}^{\prime} e^{i \gamma_{5} \theta} \quad, \quad \psi_{l}=e^{i \gamma_{5} \theta} \chi_{l}^{\prime} .
$$

With this choice, the Jacobian of this chiral transformation equals unity. If we integrate over the field $\theta(x)$ in the path integral, the resulting generating functional then takes the form

$$
\begin{aligned}
Z_{\text {ext }}[A] & =\int d[\theta] d[\bar{\chi}, \chi] \prod_{l} d\left[\bar{\chi}_{l}^{\prime}, \chi_{l}^{\prime}\right] \exp \left(\frac{i}{\hbar} S_{\text {ext }}\right) \\
S_{\text {ext }} & =\int d^{2} x\left[\bar{\chi} i \not \partial_{\theta} \chi+\sum_{l} \bar{\chi}_{l}^{\prime}\left(i \not D_{\theta}-M_{l}^{\theta}\right) \chi_{l}^{\prime}\right]
\end{aligned}
$$

where $\not \phi_{\theta}=\not D+i \not \partial \theta \gamma_{5}$, and $M_{l}^{\theta}=M_{l} \exp \left(2 i \gamma_{5} \theta\right)$. Because of the redundant introduction of a new degree of freedom, the "collective field" $\theta(x)$, the theory now has a local gauge symmetry [12]. This 
gauge symmetry is simply

$$
\bar{\chi} \rightarrow \bar{\chi} e^{i \gamma_{5} \alpha} \quad, \quad \chi \rightarrow e^{i \gamma_{5} \alpha} \psi \quad, \quad \theta \rightarrow \theta-\alpha,
$$

supplemented with the analogous transformations of the regulator fields. Now, however, the regulator fields are not the standard ones of a Pauli-Villars regularization that corresponds to the Dirac operator $i \not \phi_{\theta}-M_{l}$. In order to arrive at the standard regulator expressions one can choose to redefine the regulator fields according to

$$
\bar{\chi}_{l}^{\prime}=\bar{\chi}_{l} \quad, \quad \chi_{l}^{\prime}=\left(i \not D_{\theta}-M_{l}^{\theta}\right)^{-1}\left(i \not D_{\theta}-M_{l}\right) \chi_{l}
$$

This transformation has a non-trivial Jacobian, but it is a computable Jacobian since it only involves a ratio of determinants. We can thus write

$$
\begin{aligned}
Z_{\text {ext }}[A] & =\int d[\theta] d[\bar{\chi}, \chi] \prod_{l} d\left[\bar{\chi}_{l}, \chi_{l}\right] \exp \left(\frac{i}{\hbar} S+i \mathcal{M}_{1}\right) \\
S & =\int d^{2} x\left[\bar{\chi} i \not \phi_{\theta} \chi+\sum_{l} \bar{\chi}_{l}\left(i \not D_{\theta}-M_{l}\right) \chi_{l}\right] \\
\mathcal{M}_{1} & =-i \ln \operatorname{det} \prod_{l}\left(\frac{i \not_{\theta}-M_{l}}{i \not_{\theta}-M_{l}^{\theta}}\right)^{c_{l}}
\end{aligned}
$$

Here, $c_{l}$ are the coefficients introduced in (6). The term $M_{1}$ is the exponentiated Jacobian. It includes terms that vanish in the limit of infinite regulator masses. The gauge symmetry (10) is now supplemented by a modified transformation of the regulator fields, which follows from (11):

$$
\begin{aligned}
& \bar{\chi}_{l} \rightarrow \bar{\chi}_{l} e^{i \alpha \gamma_{5}} \\
& \chi_{l} \rightarrow e^{i \alpha \gamma_{5}}\left(i \not D_{\theta}-M_{l}^{\alpha}\right)^{-1}\left(i \not D_{\theta}-M_{l}\right) \chi_{l} .
\end{aligned}
$$

Obviously, these transformations compensate for those shifts of $\theta$ that couple to the regulator fields. In addition, the Jacobian of this transformation cancels the modification of $M_{1}$ due to the shift of $\theta$. We are now in a position to establish the BRST-transformation rules of all fields. As usual, we replace the parameter field $\alpha(x)$ in eqs. (10) and (13) - now considered infinitesimal - by the ghost field $c(x)$. In addition, we use the standard transformation rules for the auxiliary fields:

$$
\begin{aligned}
\delta \bar{\chi} & =i \bar{\chi} \gamma_{5} c \\
\delta \chi & =-i c \gamma_{5} \chi \\
\delta \theta & =-c \\
\delta \bar{\chi}_{l} & =i \bar{\chi} \gamma_{5} c \\
\delta \chi_{l} & =-\left(i c \gamma_{5}+\left(i D_{\theta}-M_{l}\right)^{-1} 2 i M_{l} c \gamma_{5}\right) \chi_{l} \\
\delta \bar{c} & =b \\
\delta c & =\delta b=0
\end{aligned}
$$

A straightforward calculation shows that these transformations are nilpotent, i.e., $\delta^{2}=0$. We may now fix a gauge by adding the BRST-variation $\delta \Psi_{R}$ of a properly regularized gauge fermion $\Psi_{R}$. To see that highly unusual gauges can be reached in this manner, let us follow a simplified version of the example given in ref. [5]. The most interesting gauge is the "bosonization gauge". In $(1+1)$ dimensions such a gauge can be found in the present context by eliminating the divergence of the axial fermionic current. [3 Note that we are not trying to gauge-fix the original fermionic degrees of

\footnotetext{
${ }^{3}$ This ignores the gauge fixing of the zero mode corresponding to constants shifts of $\theta$. As we use the example for illustration only, we shall ignore this subtlety here. The correct treatment of the zero mode can be found in ref. [5].
} 
freedom away (an impossible task, since they have physical significance), only the chirally rotated fermionic degrees of freedom. In this way we can trade fermionic degrees of freedom with bosonic ones, choosing to let the original physical fermionic currents be described by bosonic expressions. It is thus possible to gauge-fix the divergence of the chirally rotated fermionic axial current to zero. The crucial incredient is of course that we restrict ourselves to properly regularized expressions. For that reason we choose the corresponding gauge fermion $\Psi_{R}$ to be

$$
\Psi_{R}=\bar{c} \partial_{\mu}\left(\bar{\chi} \gamma^{\mu} \gamma_{5} \chi+\sum_{l} \bar{\chi}_{l} \gamma^{\mu} \gamma_{5} \chi_{l}\right)
$$

With this, the generating functional becomes

$$
\begin{aligned}
Z[A]= & \int d[\theta] d[\bar{\chi}, \chi] \prod_{l} d\left[\bar{\chi}_{l}, \chi_{l}\right] d[b] d[\bar{c}, c] \exp \left(\frac{i}{\hbar} S+i \mathcal{M}_{1}+i \delta \Psi_{R}\right) \\
S= & \int d^{2} x\left[\bar{\chi} i \not \phi_{\theta} \chi+\sum_{l} \bar{\chi}_{l}\left(i \not \phi_{\theta}-M_{l}\right) \chi_{l}\right] \\
\mathcal{M}_{1}= & -i \ln \operatorname{det} \prod_{l}\left(\frac{i \not_{\theta}-M_{l}}{i \not D_{\theta}-M_{l}^{\theta}}\right)^{c_{l}} \\
\delta \Psi_{R}= & b \partial_{\mu}\left(\bar{\chi} \gamma^{\mu} \gamma_{5} \chi+\sum_{l} \bar{\chi}_{l} \gamma^{\mu} \gamma_{5} \chi_{l}\right) \\
& +\bar{c} \partial_{\mu} \sum_{l} \bar{\chi}_{l} \gamma^{\mu} \gamma_{5}\left(i D_{\theta}-M_{l}\right)^{-1} 2 i M_{l} \gamma_{5} \chi_{l} c
\end{aligned}
$$

Note the crucial rôle played by the regulator fields in the gauge fixing proposed in eq. (15). The part $\partial_{\mu} \bar{\chi} \gamma^{\mu} \gamma_{5} \chi$ is BRST invariant by itself, and as such of course is not a suitable function to gauge-fix on. Indeed, if we had just kept that term, the gauge fixing would consist of only $b \partial_{\mu} \bar{\chi} \gamma^{\mu} \gamma_{5} \chi$, with no ghost term. Such a term is also all one would be left with if one naively applied the formulation in which the regulator fields have been integrated out from the beginning. Although naively BRST invariant, it would be incorrect for a variety of reasons, as will be seen below. The regulator fields are in eq. (15) the only fields responsible for providing the correct ghost term. The structure of $\mathcal{M}_{1}$ and the ghost term becomes more enlightened if we apply an expansion in inverse powers of the cutoff $\Lambda$. For $\mathcal{M}_{1}$ this yields

$$
\mathcal{M}_{1}=\int d^{2} x\left[\frac{1}{2 \pi} \partial_{\mu} \theta \partial^{\mu} \theta-\frac{1}{\pi} A_{\mu} \partial^{\mu} \theta+\frac{\kappa_{-2}}{12 \pi \Lambda^{2}} \partial^{2} \theta \partial^{2} \theta-\frac{\kappa_{-2}}{6 \pi \Lambda^{2}} \partial_{\nu} A_{\mu} \partial_{\nu} \partial_{\mu} \theta+\mathcal{O}\left(\Lambda^{-4}\right)\right]
$$

with

$$
\kappa_{-2}=\sum_{l} \frac{c_{l}}{k_{l}^{2}}
$$

Obviously, $\mathcal{M}_{1}$ not only provides a kinetic term for the new field $\theta$ but also higher derivative terms. These terms are responsible for a regularized perturbative $\theta$-propagator [5]. In addition, there are terms with higher power s of $\partial_{\mu} \theta$ and the external source $A_{\mu}$; it is a special feature of 2 dimensions that these occur only at order $\mathcal{O}\left(\Lambda^{-4}\right)$. Usually, one treates the regularization as part of the measure. This means that for any step in the abovementioned procedure one has to adjust the regulator to the fermionic part and integrate out the regulator fields. For the ghost term in (16) we can do this performing a linked cluster expansion for the regulator fields. To leading order in $1 / \Lambda$ we get

$$
\bar{c}\left(-\frac{1}{\pi} \partial^{2}+\frac{\kappa_{-2}}{6 \pi \Lambda^{2}}\left(\partial^{2}\right)^{2}+\ldots\right) c+\ldots
$$


The remaining terms are all of $\mathcal{O}\left(\Lambda^{-2}\right)$. They depend on higher powers in $A_{\mu}, \partial_{\mu} \theta$ and $\partial_{\mu} b$. In addition, there are also terms involving higher powers of the ghost fields; they are due to higher correlation functions of $\sum_{l} \bar{\chi}_{l} \gamma^{\mu} \gamma_{5}\left(i \not \phi_{\theta}-M_{l}\right)^{-1} 2 i M_{l} \gamma_{5} \chi_{l}$ occuring in the linked cluster expansion. But they are of higher order in $\hbar$, and should hence be ignored in this one-loop treatment. At leading order in $1 / \Lambda$ the gauge (15) coincides with the bosonization gauge of ref. [5]. However, it may also have become clear that including the regulator fields implicitly, i.e. as part of the measure, a gauge fixing procedure for finite cutoff is far more involved. In particular, the higher order terms introducing couplings between the ghost themselves, and between the ghosts and the field $b$, are difficult to interpret in standard Faddeev-Popov or BRST language. Let us now take a closer look at the gauge constraint (15). Obviously, we are removing any occurrences of the divergence of the regularized fermionic axial current in any correlation function. What then describes the divergence of the original regularized axial current $J_{5}^{\mu}$ in terms of the fields $\bar{\psi}, \psi$ and its regulators? Recall that

$$
\left\langle\partial_{\mu} J_{5}^{\mu}\left(x_{1}\right) \cdots \partial_{\nu} J_{5}^{\nu}\left(x_{n}\right)\right\rangle=\left[\frac{\delta^{n} Z[\varphi]}{\delta \varphi\left(x_{1}\right) \cdot \delta \varphi\left(x_{n}\right)}\right]
$$

where $A_{\mu}=\partial_{\mu} \varphi+\epsilon_{\mu \nu} \sigma$, and where $\sigma$ has been removed by a fermionic phase rotation (since we assume that our regulator preserves vector symmetry). Shifting $b$ by $\varphi$ decouples the fermions $\bar{\chi}, \chi$ and its regulators from $\varphi$. Functional derivatives with respect to $\varphi$ then get contributions only from $M_{1}$ and from the ghost term. This means that for the present gauge we describe the divergence of the original axial current by the purely bosonic expression $-(1 / \pi) \partial^{2} \theta$, up to terms corresponding to higher derivatives and powers of $\theta$, suppressed by inverse powers of the cutoff. So to leading order in $1 / \Lambda$, we precisely get the sought-for identification. Note that for finite cut-off $\Lambda$ we are left among others with highly unusual ghost terms, which could not straightforwardly have been derived by a Faddeev-Popov method.

3. We now translate the main steps of the previous section into the language of (regularised) Batalin-Vilkovisky (BV) Lagrangian quantisation [4]. Since no detailed introduction to this scheme will be given here, readers who are not familiar with it may skip this section. Introductions to the BV quantisation scheme can be found in [13, 14, 15, 16].

In the BV scheme one associates with every field $\phi^{A}$ an antifield $\phi_{A}^{*}$ of opposite Grassmann parity. The antibracket of two function(al)s $F$ and $G$ of the fields and antifields, is defined by

$$
(F, G)=\frac{\delta^{r} F}{\delta \phi^{A}} \frac{\delta^{l} G}{\delta \phi_{A}^{*}}-\frac{\delta^{r} F}{\delta \phi_{A}^{*}} \frac{\delta^{l} G}{\delta \phi^{A}} .
$$

Canonical transformations within the antibracket are generated by a Grassmann odd function $F\left(\phi, \phi^{*^{\prime}}\right)$ of ghost $t^{\text {number }-1}$. Canonical transformation may produce a non-trivial Jacobian.

The classical action and the BRST transformation rules are brought together in the extended action $S$. The most general form needed for our present purposes is

$$
S=S_{0}(\phi)+\phi_{A}^{*} \cdot \delta \phi^{A} .
$$

The extended action satisfies the classical Master Equation $(S, S)=0$.

When considering a PV regularised theory, it is convenient to copy the set-up one has for the fields and antifields to the PV fields, i.e.:

$$
(F, G)=\frac{\delta^{r} F}{\delta \phi^{A}} \frac{\delta^{l} G}{\delta \phi_{A}^{*}}-\frac{\delta^{r} F}{\delta \phi_{A}^{*}} \frac{\delta^{l} G}{\delta \phi^{A}}+\frac{\delta^{r} F}{\delta \phi_{P V}^{A}} \frac{\delta^{l} G}{\delta \phi_{A, P V}^{*}}-\frac{\delta^{r} F}{\delta \phi_{A, P V}^{*}} \frac{\delta^{l} G}{\delta \phi_{P V}^{A}} .
$$

\footnotetext{
${ }^{4}$ The ghost number of the field and the ghost number of the antifield are related by $\operatorname{gh}\left(\phi^{A}\right)+\operatorname{gh}\left(\phi_{A}^{*}\right)=-1$.
} 
Our starting point is the regularised action of fermions coupled to an external gauge field given in (5). One can imagine enlarging the degrees of freedom in the theory with a scalar field $\theta$. Since the action $S_{0}$ does not depend on $\theta$, there is a shift gauge symmetry $\delta \theta=\epsilon$. Introducing a ghost field $c$ for this symmetry, one obtains the extended action

$$
S=\int d^{2} x\left[\bar{\psi} i \not D \psi+\sum_{l} \bar{\psi}_{l}\left(i D-M_{l}\right) \psi_{l}+\theta^{*} c\right],
$$

which obviously satisfies the classical Master Equation.

The chiral rotation from the $\psi$ to the $\chi$ variables can be implemented by a canonical transformation generated by

$$
F_{1}=\theta^{*^{\prime}} \theta+c^{*^{\prime}} c+\bar{\psi} e^{-i \gamma_{5} \theta} \bar{\chi}^{*}+\chi^{*} e^{-i \gamma_{5} \theta} \psi+\bar{\psi}_{l} e^{-i \gamma_{5} \theta} \bar{\chi}_{l}^{*}+\chi_{l}^{*} e^{-i \gamma_{5} \theta} \psi_{l} .
$$

This generating fermion produces the following transformations of the fields, antifields and their regularisation counterparts (we suppress spinor indices):

$$
\begin{array}{cc}
\chi=\frac{\delta F_{1}}{\delta \chi^{*}}=e^{-i \gamma_{5} \theta} \psi & \chi_{l}=\frac{\delta F_{1}}{\delta \chi_{l}^{*}}=e^{-i \gamma_{5} \theta} \psi_{l} \\
\bar{\chi}=\frac{\delta F_{1}}{\delta \bar{\chi}^{*}}=\bar{\psi} e^{-i \gamma_{5} \theta} & \bar{\chi}_{l}=\frac{\delta F_{1}}{\delta \bar{\chi}_{l}^{*}}=\bar{\psi}_{l} e^{-i \gamma_{5} \theta} \\
\theta^{\prime}=\frac{\delta F_{1}}{\delta \theta^{*^{\prime}}}=\theta & c^{\prime}=\frac{\delta F_{1}}{\delta c^{*^{\prime}}}=c,
\end{array}
$$

which corresponds to the transformation (7) and (8).

However, (25) also determines the transformation of the antifields. In particular, one finds

$$
\theta^{*}=\frac{\delta F_{1}}{\delta \theta}=\theta^{*^{\prime}}-i \bar{\chi} \gamma_{5} \bar{\chi}^{*}-i \chi^{*} \gamma_{5} \chi-i \bar{\chi}_{l} \gamma_{5} \bar{\chi}_{l}^{*}-i \chi_{l}^{*} \gamma_{5} \chi_{l}
$$

Here the transformation rules of the fields have already been used. The extended action (24) transforms into (dropping the primes on $\theta$ and $c$ )

$$
\begin{aligned}
S_{1}= & \int d^{2} x\left[\bar{\chi} i D_{\theta} \chi+\sum_{l} \bar{\chi}_{l}\left(i D_{\theta}-M_{l}^{\theta}\right) \chi_{l}+\theta^{*} c\right. \\
& \left.-i \bar{\chi} \gamma_{5} c \bar{\chi}^{*}+i \chi^{*} \gamma_{5} c \chi-i \bar{\chi}_{l} \gamma_{5} c \bar{\chi}_{l}^{*}+i \chi_{l}^{*} \gamma_{5} c \chi_{l}\right] .
\end{aligned}
$$

This canonical transformation does not produce any Jacobian.

As was pointed out above, after the canonical transformation, the classical action $S_{1}\left(\phi, \phi^{*}=\right.$ 0 ) has gauge symmetry (10). Notice that by implementing the chiral rotation as a canonical transformation, one immediately reads off the correct BRST rules corresponding to this symmetry from the transformed extended action (28).

The next step is to make sure that the regulator fields get the correct mass term $-\bar{\chi}_{l} M \chi_{l}$ instead of the mass term $-\bar{\chi}_{l} M^{\theta} \chi_{l}$ that resulted from the chiral rotation. Again this can be done using a canonical transformation, where the appropriate generating fermion is now

$$
F_{2}=\mathbb{1}+\mu_{l}^{*} \frac{1}{i D_{\theta}-M_{l}}\left(M_{l}-M_{l}^{\theta}\right) \chi_{l} .
$$

The only fields that change under this canonical transformation are $\chi_{l}, \chi_{l}^{*}$ and $\theta^{*}$ :

$$
\begin{aligned}
& \mu_{l}=\frac{\delta F_{2}}{\delta \mu_{l}^{*}}=\chi_{l}+\frac{1}{i D_{\theta}-M_{l}}\left(M_{l}-M_{l}^{\theta}\right) \chi_{l}=\frac{1}{i D_{\theta}-M_{l}}\left(i D_{\theta}-M_{l}^{\theta}\right) \chi_{l} \\
& \chi_{l}^{*}=\frac{\delta F_{2}}{\delta \chi_{l}}=\mu_{l}^{*}+\mu_{l}^{*} \frac{1}{i D_{\theta}-M_{l}}\left(M_{l}-M_{l}^{\theta}\right)=\mu_{l}^{*} \frac{1}{i D_{\theta}-M_{l}}\left(i D_{\theta}-M_{l}^{\theta}\right) \\
& \theta^{*}=\frac{\delta F_{2}}{\delta \theta}=\theta^{*^{\prime}}+\frac{\delta^{r}}{\delta \theta}\left[\mu_{l}^{*} \frac{1}{i D_{\theta}-M_{l}}\left(M_{l}-M_{l}^{\theta}\right) \chi_{l}\right] .
\end{aligned}
$$


The effect of this canonical transformation on the antifield independent part of the extended action is trivial, it just produces the required change from

$$
\bar{\chi}_{l}\left(i D_{\theta}-M_{l}^{\theta}\right) \chi_{l} \longrightarrow \bar{\chi}_{l}\left(i D_{\theta}-M_{l}\right) \mu_{l} .
$$

The transformation on the antifield dependent part is somewhat more complicated, and therefore we will provide some detail. The two terms in (28) that are going to change are

$$
\theta^{*} c+i \chi_{l}^{*} \gamma_{5} c \chi_{l}
$$

The second of these two terms (32) changes into

$$
T_{1}=i \mu^{*} \frac{1}{i \not D_{\theta}-M}\left(i D_{\theta}-M^{\theta}\right) \cdot \gamma_{5} c \cdot \frac{1}{i \not D_{\theta}-M^{\theta}}\left(i D_{\theta}-M\right) \mu,
$$

where the subindex $l$ labelling the different PV copies is suppressed. Let us now commute the $\gamma_{5} c$ to the left until it is just behind the antifield $\mu^{*}$. The only trick to be used is the triviality

$$
\left[\frac{1}{A}, B\right]=-\frac{1}{A}[A, B] \frac{1}{A} .
$$

One then easily finds

$$
\begin{aligned}
T_{1}= & \mu^{*} i \gamma_{5} c \mu-\mu^{*} \frac{1}{i \not D_{\theta}-M}\left(\gamma_{5} \not \partial c+2 \gamma_{5} c D_{\theta}\right) \mu \\
& +\mu^{*} \frac{1}{i D_{\theta}-M}\left(\gamma_{5} \not \partial c+2 \gamma_{5} c \not D_{\theta}\right) \frac{1}{i \not D_{\theta}-M^{\theta}}\left(i D_{\theta}-M\right) \mu .
\end{aligned}
$$

To perform the canonical transformation on the first term in (32) one has to be slightly more careful. It transforms to

$$
T_{2}=\theta^{*} c+\int d^{2} y \int d^{2} x \mu^{*}(x) \frac{\delta^{r}}{\delta \theta(y)}\left[\frac{1}{i D_{\theta}^{x}-M}\left(i D_{\theta}^{x}-M_{l}^{\theta(x)}\right)\right] \cdot \frac{1}{i D_{\theta}^{x}-M^{\theta(x)}}\left(i D_{\theta}^{x}-M\right) \mu(x) c(y) .
$$

Again the index $l$ was suppressed and we have made all hidden space-time coordinates explicit. Probably the quickest way to proceed is to use that $\partial_{\mu}^{x} \delta(x-y)=-\partial_{\mu}^{y} \delta(x-y)$. One finds

$$
\begin{aligned}
T_{2}= & \theta^{*} c+\mu^{*} \frac{1}{i D_{\theta}-M} 2 i \gamma_{5} c M \mu+\mu^{*} \frac{1}{i \not D_{\theta}-M}\left(\gamma_{5} \not \partial c+2 \gamma_{5} c D_{\theta}\right) \mu \\
& -\mu^{*} \frac{1}{i D_{\theta}-M}\left(\gamma_{5} \not \partial c+2 \gamma_{5} c D_{\theta}\right) \frac{1}{i D_{\theta}-M^{\theta}}\left(i D_{\theta}-M\right) \mu .
\end{aligned}
$$

Several terms cancel between $T_{1}$ and $T_{2}$, and one obtains

$$
\theta^{*} c+i \chi_{l}^{*} \gamma_{5} c \chi_{l} \stackrel{\text { can. traf. }}{\longrightarrow} \theta^{*} c+\chi_{l}^{*}\left(i \gamma_{5} c+\frac{1}{i D_{\theta}-M_{l}} 2 i \gamma_{5} c M_{l}\right) \chi_{l}
$$

where we have changed notation back to the $\chi$-fields. Summarising, one finds that the extended action (28) transforms into

$$
\begin{aligned}
S_{2}= & \int d^{2} x\left[\bar{\chi} i D_{\theta} \chi+\sum_{l} \bar{\chi}_{l}\left(i D_{\theta}-M_{l}\right) \chi_{l}+\theta^{*} c\right. \\
& \left.-i \bar{\chi} \gamma_{5} c \bar{\chi}^{*}+i \chi^{*} \gamma_{5} c \chi-i \bar{\chi}_{l} \gamma_{5} c \bar{\chi}_{l}^{*}+\chi_{l}^{*}\left(i \gamma_{5} c+\frac{1}{i D_{\theta}-M_{l}} 2 i \gamma_{5} c M_{l}\right) \chi_{l}\right] .
\end{aligned}
$$


The canonical transformation generated by (29) has also produced a Jacobian. As is obvious from (30), it is precisely the one shown in (12).

We now construct a so-called non-minimal extended action, $S_{2}^{n . m} .=S_{2}+\bar{c}^{*} b$, and perform a third canonical transformation generated by

$$
F_{3}=\mathbb{1}+\Psi_{R} .
$$

Since $\Psi_{R}$ only depends on fields, only the antifields will transform non-trivially under this canonical transformation, and therefore the Jacobian is 1 . The extended action changes precisely by the addition of $\delta \Psi_{R}$ of (16):

$$
S_{3}=S_{2}^{n \cdot m \cdot}+\delta \Psi_{R}
$$

At this point, one can put the antifields to zero, and go through all the steps described above. In this way we have rederived bosonization in the BV scheme. Introducing a free parameter $\Delta$ as explained below, we also rederive the generalization, smooth bosonization, in BV language.

4. The procedure we have outlined above is of course general, and in principle straightforward. The most important aspect to keep in mind is that the regulator fields must be kept explicitly in the path integral throughout, and in particular when one is gauge-fixing (or changing gauges) by the addition of BRST-exact terms. The second requirement is the natural one of always manipulating properly regularized expressions.

In ref. [7] a more convenient way of achieving the same kind of "quantum mechanical gauge" that we have derived above was described. It hinges rather crucially on the situation peculiar to the bosonization example discussed here: the collective field $\theta(x)$ which is responsible for the new gauge symmetry does not require additional regulator fields. ${ }^{5}$ However, some aspects have more general validity, and since it very nicely elucidates the issue of gauge fixing in the presence of regularization, we shall now outline it.

Let us first state in more clear terms which gauge we finally wish to achieve. In the bosonization gauge of smooth bosonization [5], the idea is to find a gauge such that the physical (and gauge invariant) axial current $\psi \gamma^{\mu} \gamma_{5} \psi$ is described entirely by a bosonic expression. This is set up to hold in the full generating functional (and of course then also off mass shell).

As we have shown above, this is difficult (but possible) because it involves gauge-fixing away the chirally rotated fermionic axial current $\partial_{\mu} \bar{\chi} \gamma^{\mu} \gamma_{5} \chi$, and in its unregularized form this object is gauge (or BRST) invariant. One way to cure the problem is, as we have demonstrated above, to keep explicitly the regulator fields in the path integral. But suppose we adhere to the formulation in which the regulator fields have already been integrated out, leaving a non-trivial regularized $\bar{\chi}, \chi$-measure: how do we proceed? We shall now show that a short-cut exists in this formulation. The idea is to first gauge-fix on an entirely unproblematic function, and then shift the axial source in a BRST invariant manner so as to finally reach the non-trivial gauge we seek. That such a procedure is possible is due to the fact that the gauge symmetry we are considering arises from a field redefinition (a chiral rotation) of exactly the same kind as the gauge transformation itself. The Jacobian we obtain from the field redefinition is then in form completely equivalent to the Jacobian we subsequently get under BRST transformations.

When only the $\bar{\psi}, \psi$-measure contains the regularization, already the first step (eq. (7)) above is modified. The Jacobian of the field redefinition is now non-trivial, and the analogue of eq. (9) therefore contains a quantum correction:

$$
Z_{\text {ext }}=\int d[\theta] d[\bar{\chi}, \chi]_{\Lambda} \exp \left(\frac{i}{\hbar} S_{\text {ext }}\right)
$$

\footnotetext{
${ }^{5}$ Intuitively this is easy to understand, since the whole path integral is regularized to begin with, and redefinitions of the fields do not affect this overall regularization. This holds even if we integrate over the additional collective fields, because the gauge-fixing is precisely removing the new redundant propagating degree of freedom.
} 


$$
S_{\text {ext }}=\int d^{2} x\left[\bar{\chi} i \not D_{\theta} \chi+i \hbar \mathcal{M}_{1}^{(\Lambda)}\left(\theta, A_{\mu}\right)\right]
$$

Here, the Jacobian $\mathcal{M}_{1}^{(\Lambda)}$ can be expressed in terms of an infinite expansion in inverse powers of the ultraviolet cut-off $\Lambda$ :

$$
\begin{aligned}
\mathcal{M}_{1}(\Lambda) & =-i \ln \operatorname{det} \prod_{l}\left(\frac{i \not D_{\theta}-M_{l}}{i \not D_{\theta}-M_{l}^{\theta}}\right)^{c_{l}} \\
& =\int d^{2} x\left[\frac{1}{2 \pi} \partial_{\mu} \theta \partial^{\mu} \theta-\frac{1}{\pi} A_{\mu} \partial^{\mu} \theta+\frac{\kappa_{-2}}{12 \pi \Lambda^{2}} \partial^{2} \theta \partial^{2} \theta-\frac{\kappa_{-2}}{6 \pi \Lambda^{2}} \partial_{\nu} A_{\mu} \partial_{\nu} \partial_{\mu} \theta+\mathcal{O}\left(\Lambda^{-4}\right)\right]
\end{aligned}
$$

The cut-off itself is related to the Pauli-Villars regulator masses as defined in (6).

The idea of ref. [7] is now to first gauge-fix in the more simple $\theta$-sector. This may sound paradoxical, since in the bosonization gauge we precisely wish to retain fully the $\theta$-field. However, the next step consists in shifting the external source $A_{\mu}$ by the derivative of the Lagrangian multiplier, $\partial_{\mu} b$. As we will see, this can be done in such a way as to precisely cancel the effect of gauge fixing on $\theta$. We thus first add

$$
-\frac{i \hbar}{\pi} \delta\left(\bar{c} \partial^{2} \theta\right)=-\frac{i \hbar}{\pi} b \partial^{2} \theta+\frac{i \hbar}{\pi} \bar{c} \partial^{2} c
$$

to the action. I This is a correct gauge fixing term for setting $\partial^{2} \theta=0$. Next, we shift the source: $A_{\mu} \rightarrow A_{\mu}+\partial_{\mu} b$. This is a BRST-invariant procedure because $A_{\mu}$ couples only to a term that has vanishing BRST-variation, and $b$ itself is neutral under BRST transformations. The result is an action of the form

$$
\begin{aligned}
S_{\text {ext }} & =\int d^{2} x\left[\bar{\chi} i \not \partial_{\theta+b} \chi+i \hbar \mathcal{M}_{1}\left(A_{\mu} \rightarrow A_{\mu}+\partial_{\mu} b\right)-\frac{i \hbar}{\pi} b \partial^{2} \theta+\frac{i \hbar}{\pi} \bar{c} \partial^{2} c\right] \\
& =\int d^{2} x\left[\bar{\chi} i \not \partial_{\theta+b} \chi+i \hbar \ldots+\frac{i \hbar}{\pi} \bar{c} \partial^{2} c\right]
\end{aligned}
$$

Note that there is a term $b \partial^{2} \theta$ from $\mathcal{M}_{1}$ (see (17)) which just cancels the gauge fixing term we started with. With this modification, we precisely identify

$$
\partial_{\mu} J_{5}^{\mu} \sim-1 / \pi \partial^{2} \theta .
$$

This can be seen easily by shifting $b$ by $\varphi$ with $A_{\mu}=\partial_{\mu} \varphi$. This shift removes all occurrences of the external source $\varphi$ except for its coupling to $\partial^{2} \theta$.

A rephrasing of this procedure in the limit of infinite cutoff has recently been discussed in ref. [17]. Duality transformations between bosons and fermions in $(1+1)$ dimensions also effectively rely on gauge fixings in sectors that are free of complications due to regularization [18].

The biggest advantage of the procedure described above is that in general the precise content of the gauge fixed theory is completely known. It gives an exact specification of the generating functional in terms of the degrees of freedom we choose, and hence all relevant Green functions computed in terms of those degrees of freedom. Let us finally formulate the procedure in a more general manner.

We start with a functional integral governed by an action $S$ parametrized as follows:

$$
S\left[\phi^{A}, A_{r}\right]+\hbar \mathcal{M}_{1}\left[\phi^{A}, A_{r}\right]
$$

where $\phi^{A}$ and $A_{r}$ denote the various fields and external sources. The theory has a gauge symmetry

$$
\delta \phi^{A}=R_{i}^{A}[\phi] \epsilon^{i}
$$

${ }^{6}$ Note the explicit factor of $\hbar$ here. 
such that the Jacobian of this transformation is cancelled by the variation of $M_{1}$. The sources $A_{r}$ are chosen to couple only to gauge invariant operators $\mathcal{O}_{r}$. The generating functional is thus gauge invariant even for non-vanishing sources.

Suppose that a subset of the fields $\phi^{A}$, denoted by $\phi^{i}$, transforms under this gauge transformation with unit Jacobian. Furthermore, suppose we wish to find the gauge that identifies these fields $\phi^{i}$ with operators $\mathcal{O}^{i}$ that couple to the sources $A_{i}$; this, of course, implies that there are couplings $A_{i} \phi^{i}$. We can achieve this by first gauge fixing the fields $\phi^{i}$ to zero by adding

$$
\delta \Psi=\delta\left(\bar{c}_{i} \phi^{i}\right)=b_{i} \phi^{i}-\bar{c}_{i} R_{j}^{i} c^{j} .
$$

Next, shift the source $A_{i} \rightarrow A_{i}-b_{i}$, and subsequently shift $b_{i} \rightarrow b_{i}+A_{i}$. The source $A_{i}$ then couples only to $\phi^{i}$, i.e., we have reached the gauge in which the (gauge invariant) operator $\mathcal{O}^{i}$ is represented by the (gauge dependent) field $\phi^{i}$. A gauge aiming at identifying $\phi^{i}$ with only a given fraction $\Delta$ of the operator $\mathcal{O}^{i}$ can be obtained by replacing the shift of $A_{i}$ above by $A_{i} \rightarrow A_{i}-\Delta b_{i}$. In smooth bosonization, $\Delta$ is the parameter by means of which one can interpolate smoothly between a bosonic and a fermionic gauge.

Acknowledgment: The work of FDJ was supported by the Human Capital and Mobility Programme through a network on Constrained Dynamical Systems.

\section{References}

[1] F. De Jonghe, R. Siebelink, W. Troost, S. Vandoren, P. van Nieuwenhuizen and A. Van Proeyen, Phys. Lett. B289 (1992) 354.

[2] L.D. Faddeev and V.N. Popov, Phys. Lett. 25B (1967) 29.

[3] T. Kugo and I. Ojima, Prog. Theor. Phys. 60 (1978) 1869.

[4] I.A. Batalin and G.A. Vilkovisky, Phys. Lett. 102B (1981) 27; ibid. 120B (1983); Phys. Rev. D28 (1983) 2567 [E: D30 (1984) 508].

[5] P.H. Damgaard, H.B. Nielsen and R. Sollacher, Nucl. Phys. B385 (1992) 227; Phys. Lett. B296 (1992) 132.

[6] P.H. Damgaard, H.B. Nielsen and R. Sollacher, Nucl. Phys. B414 (1994) 541.

[7] P.H. Damgaard and R. Sollacher, Phys. Lett. B322 (1994) 131; Nucl. Phys. B433 (1995) 671.

[8] A. Niemi, Ann. Phys.(N.Y.) 187 (1988) 369.

[9] K. Fujikawa, Phys. Rev. D21 (1980) 2848.

[10] A. Diaz, W. Troost, P. van Nieuwenhuizen and A. Van Proeyen, Int. J. Mod. Phys. A4 (1989) 3959 .

[11] R.D. Ball, Phys. Rep. 182 (1989) 1.

[12] J. Alfaro and P.H. Damgaard, Ann. Phys.(N.Y.) 202 (1990) 398. 
[13] F. De Jonghe, PhD. thesis K.U. Leuven 1994, hep-th/9403143.

[14] W. Troost and A. Van Proeyen, An introduction to Batalin-Vilkovisky Lagrangian Quantisation, Leuven Notes in Math. Theor. Phys., in preparation.

[15] J. Gomis, J. Paris and S. Samuel, hep-th/9412228.

[16] M. Henneaux and C. Teitelboim, Quantisation of gauge systems, Princeton University Press, 1992.

[17] A.N. Theron, F.A. Schaposnik, F.G. Scholtz and H.B. Geyer, Nucl. Phys. B437 (1995) 187.

[18] C.P. Burgess and F. Quevedo, Phys. Lett. B329 (1994) 457; Nucl. Phys. B421 (1994) 373.

C.P. Burgess, A. Lütken and F. Quevedo, Phys. Lett. B336 (1994) 18.

J.L Cortés, E. Rivas and L. Velazquez, preprint DFTUZ95-10, hep-th/9503194. 